\title{
Racines artistiques et imaginaires extra-terrestres
}

\section{Artistic roots and extraterrestrial imaginations}

\author{
Sylvie Dallet ${ }^{1}$ \\ ${ }^{1}$ professeure des universités (Université Gustave Eiffel, Arts), directrice de recherches (CHCSC, Université de Paris \\ Saclay), présidente de l'Institut Charles Cros
}

RÉSUMÉ. L'exploration d'un imaginaire extra-terrestre suppose une curiosité pour les formes végétales plutôt que les animales, qui sont longtemps restées les modèles préférés de peintres. L'artiste contemporain, détaché des canons de la représentation historique, peut, plus facilement que par le passé, exercer sa puissance créative de façon analogique, embrassant un vivant complexe et bourgeonnant. La redécouverte des formes et des expressions végétales, rondes, sinueuses, hybrides et foisonnantes offre, par ce retour aux formes archaïques, génésiques ou rêvées, des pistes à l'anticipation de la vie cosmique.

ABSTRACT. The exploration of an extraterrestrial imaginary presupposes a curiosity for plant forms rather than animals, which have long remained the preferred models of painters. The contemporary artist, detached from the canons of historical representation, can, more easily than in the past, exercise his creative power analogically, embracing a living complex and budding. The rediscovery of vegetal forms and expressions, round, sinuous, hybrid and abundant, offers, by this return to archaic, reproductive or dreamed forms, paths to the anticipation of cosmic life.

MOTS-CLÉS. Arborescence, Art, Cocon, Graine, Imaginaire extra-terrestre, Racines, Règne végétal.

KEYWORDS. Tree, Art, Cocoon, Seed, Extraterrestrial imagination, Roots, Plant kingdom.

Répondre à mon ami Georges Chapouthier suppose des connaissances scientifiques qui me sont (à l' instant où j'écris) bien étrangères, malgré la matrice qu'il fournit. Cependant, par le biais d'exemples pris à la périphérie, de mots picorés dans le texte introductif, de tous ces termes communs que l'humanité se partage, je peux, par une méthode volontairement diséminée, essayer de répondre à l'exploration d'un "imaginaire extraterrestre".

Ma méthode est celle de l'analogie et des bonds qu'elle suppose vers le plus étrange. Comprendre et agir par la ressemblance est l'échelle qui permet d'entrer dans des connaissances plus complexes, des formes multiples que l'imagination reçoit dès qu'elle accepte ses rêves. Sortir de la caverne suppose une progression, marche par marche, guidée à l'ouïe par des rythmes musicaux différents, dans un accouchement de soi qui ne peut se partager. Le mythe d'Orphée et l'apologue de Platon ont lointainement partie liées, de même que, pour la Bible, l'échelle de Jacob que les anges gravissent pour redescendre sans cesse. Nous sommes liés aux profondeurs, au cosmos et aux astres par un cordon secret que les formes diverses du vivant et les couleurs chatoyantes des fleurs peuvent nous laisser comprendre. Seule la rapidité de la reconnaissance diffère et c'est dans cette fulgurance, ou cette lenteur différée, que l'art offre son expertise singulière. L'artiste en effet accepte (parce qu'il y est soumis) la fulgurance, l'urgence, la transe qui fait coïncider sa perception à un être au monde.

Répondre à mon ami suppose, en profondeur, une gravitation littéraire tout autant qu'artistique. Le cordon et le tournis, comme le fruit d'une fronde qui claque sur la tempe du géant scientifique, Goliath réinventé. David l'artiste, David le créateur en survie, utilise son lacet et un caillou de sa chaussure. Créer est un acte sous contrainte qui s'appuie sur de l'existant pour le faire dériver. La volonté intervient assez peu dans des processus où l'inconscient et surtout la confiance en un "ensoi" demeurent primordiaux. La genèse d'une œuvre peut s'expliquer de multiples façons, de même que l'on tente d'expliquer la naissance d'une étoile : il manque toujours quelque chose de secret, le Rosebud qu'Orson Welles mit en scène en 1946 pour tenter de comprendre la fin d'un magnat de la presse. 
Par ailleurs, pour reprendre la suggestion de Georges Chapouthier sur les invariants de la formation des espèces, l'histoire du monde humain qui oscille entre la conservation et la transformation, porte les traces d'un imaginaire extraterrestre : l'histoire des arts (et des pensées que les arts mettent en forme) témoigne d'époques inventives où l'humanité a inventé des formes symboliques qui extrapolaient vers le futur. Star Trek, la saga américaine du vaisseau Enterprise foisonne des années 1966 (759 épisodes)- aux années 2000 (96 épisodes de 42 minutes) d'experiences qui relient les extra-terrestres à l'aventure galactique. Pour donner au cinéma américain un seul équivalent soviétique, le film muet Aelita de Yakov Protazanov, réalisé en 1924, donne une vision inversée de la découverte des formes martiennes, inspiré d'une œuvre de Tolstoï. La scène finale d'Aelita montre un couple réconcilié qui brûle dans la cheminée de son appartement terrestre les plans d'une fusée. La cheminée comme l'échelle participe de l'imaginaire de l'élévation, de l'évasion, de la fumée des formes possibles. L'imaginaire extra-terrestre puise son réseau racinaire dans le rêve et l'effort absolu de le transcrire cette vision sous une forme non écrite. L'écriture en effet depuis la protohistoire des hiéroglyphes aux formes figuratives (et parfois mutantes ou thériantropes ${ }^{1}$ ), s'est éloignée des représentations animales pour se rapprocher des nombres. L'Histoire situe cette mutation à la construction des Pyramides, où les ouvriers phéniciens commencèrent à communiquer entre eux par une écriture consonantique et non pictographiques. À rebours, l'acceptation depuis un siècle de «l'art des fous ", dit aujourd'hui « art brut », procède d'une lente reconquête des formes efflorescentes du vivant, animal et végétal. L'œuvre d'art est toujours une projection vers le futur dans la mesure où le présent accepte de la faire exister.

L'œuvre de création continue sur des voies insoupçonnées, en suspension et hors de la vue. Il ne s'agit plus de représenter des anges, créatures extra-terrestres, sans pieds ni mains, dans des formes convenues où seule la lumière permet l'originalité figurative. Les mises en scènes sur les corps et le mystère des organes reprennent de l'écoute auprès des scientifiques comme auprès des artistes contemporains, dans l'infiniment petit des microbiotes et des bactéries. Pour exemple, le couple Marion Laval-Jeantet et Benoit Mangin (Art orienté Objet) se passionne pour le microbiote, ces milliards de bactéries qui peuplent notre corps, notre peau, nos intestins. Le corps est une jungle comparable aux forêts du monde qui coopèrent dans le dessein de la survie et du déploiement. Ces «étrangers microscopiques » qui nous font vivre (ou nous parasitent), ont eu droit à une étonante exposition analogique en 2016 (Microbiota, commissaire d'expostion Amélie Lavin) au musée des Beaux-Arts de Dole, ville natale de Louis Pasteur. Peindre n'est pas disséquer : il ne s'agit plus de séquencer pour comprendre le vivant, mais de suggérer la vie qui traverse. ${ }^{2}$ Peindre n'est pas disséquer : il ne s'agit plus de séquencer pour comprendre le vivant, mais de suggérer la vie qui le traverse.

Le philosophe Baptiste Morizot choisit à la fois en 2020 d'intituler un de ses ouvrages Manières d'être vivant et de le faire postfacer par l'essayiste et écrivain de science-fiction Alain Damasio. Baptiste Morizot tente de renouer le dialogue entre une espèce qui a, naguère, fait "sécession" d'une dizaine de millions d'autres. L'espèce humaine, par les outils qu'elle promeut et les récits qu'elle tisse s'est en effet bien éloignée des animaux, même si, au gré des fables, proverbes et des apologues, elle s'y retrempe comme Antée. Des courants tels que le futurisme de Marinetti ${ }^{3}$ et dans un registre différent l'œuvre de Fernand Léger dans les années 1950 se passionnaient pour la

\footnotetext{
${ }^{1}$ Thériantrope : qualifie la métamorphose d'un humain en animal de façon totale ou partielle tel Horus à tête de faucon pour l'Égypte ou Actéon mué en cerf por la mythologie gréco-latine.

${ }^{2}$ Préface de Sylvie Dallet, "Peindre ou disséquer », pour l'essai Roussau, un ours dans le salon des Lumières, de Marie-Paule Farina, collection « Éthiques de la Création »(Harmattan/ Institut Charles Cros), 2021

${ }^{3}$ L'écrivain italien Filippo Marinetti (1876-1944) publie le Manifeste du futurisme en 1909 ; Pour lui, l'expression du futurisme refuse avec le passé, toute définition d'un "art auratique" dont l'objet serait magnifié par sa signification spirituelle.
} 
création industrielle. Plus subtilement, ils passaient du "sujet " en peinture à "l'objet" représenté comme influant directement sur le psychisme humain. La force des objets revient lentement à la force des choses et à la force des formes. Le philosophe Simondon avait déjà abordé l'influence des formes industrielles sur la pensée contemporaine. Dans sa postface, Damasio forge le mot de "pananimal" qui rejoint la société de "trace". Il aborde aussi sous le thème du "cocon" le thème de "big mother", un système de technococon où chacun est flatté dans la fluidité illusoire de sa consommation. La grande mère nourricière, la florissante Gaïa des sociétés primitives, semble par l'auteur de science-fiction concurrencée par une "big Mother" technologique et abusive, stérilisante et conformiste. À contrario, le thème du cocon dont George Chapouthier suggère la pérennité dans le vivant est un indicateur des formes du futur : le passage du ver à soie, la transformation du papillon deviennent des indicateurs d'une culture qui change. Dans les années 1960, dans cette époque que les économistes appellent les Trente Glorieuses, l'avenir artistique reflétait la systématisation industrielle : peu de vivant, un art conceptuel ou le normatif était mis en scène de façon obsessionnelle. L'architecture de masse, la ligne, la musique sérielle et l'installation exprimaient la norme d'une élite formée à penser conceptuellement sans la présence désordonnée du vivant. Aujourd'hui, ce vivant se délite et mue dans un climat anxiogène lié à l'effondrement de la biodiversité. Les formes proliférantes d'une arborescence originelle servent de bouées vers l'avenir.

Dans cette recherche des formes extra-terrestres que suggère Georges Chapouthier, la communication multimédia dénoncée par Damasio ne floute pas totalement l'avenir car la connaissance de l'Histoire apporte, a contrario, ses lumières. Nous créons en miroir du passé, sur des étapes (conscientes et inconscientes, la mémoire du monde) qui nous touchent, qui agissent comme des points d'acupuncture de notre mémoire. L'époque qui se dessine en référence de la nôtre, le Moyen-Âge, a donné des cathédrales dans une époque où les mythes, les rituels et les cérémonies semblaient des relais majeurs à la créativité artistique. Dans les églises romanes, les chapiteaux sculptés offrent aux regards des formes hybrides héritées des civilisations antérieures (Égypte, Assyrie, espaces africains, amérindiens et celtes etc...) telles que l'homme vert, la femme sirène, l'homme sanglier... Les chapiteaux hybrides dans l'art roman, surplombant le public, suggèrent à l'imagination des retours de formes multiples hors-sol, telle la résidence contemporaine menée par la plasticienne et photographe Diane Cazelles ${ }^{4}$ ou mes expériences esthétiques menées en université avec les étudiants.

Depuis quelques années en effet, Diane Cazelles propose des résidences d'été futuristes dans la chapelle castrale perchée de Saint-Ilpize (Haute-Loire). Pour exemple en 2019, la résidence d'artistes est dédiée à la faune et la flore naturelles et imaginaires de la rivière, en reconnaissance des richesses de notre patrimoine environnemental. Des créateurs pluridisciplinaires se retrouvent dans ce lieu pour travailler et créer une ou plusieurs œuvres. En 2021, le thème retenu « espèces en voie d'apparition» mobilise six artistes différents sur une semaine, qui exposent leur œuvre collective le dimanche. Le résultat de cette créativité de six jours révèle en creux l'imaginaire du Moyen Âge, une époque crépusculaire ou illuminée selon les récits, mais surtout une efflorescence des formes et des représentations qui culmine, pour exemple, dans l'œuvre de Jérôme Bosch. Par ailleurs, l'évolution de la photographie suggère également, la rencontre des éléments mus par un télescopage de nature différente.

\footnotetext{
${ }^{4}$ Ancienne élève des Beaux-Arts et de l'École du Louvre, Diane Cazelles est plasticienne, journaliste et photographe qui se partage entre la Haute-Loire et la Région Parisienne.
} 


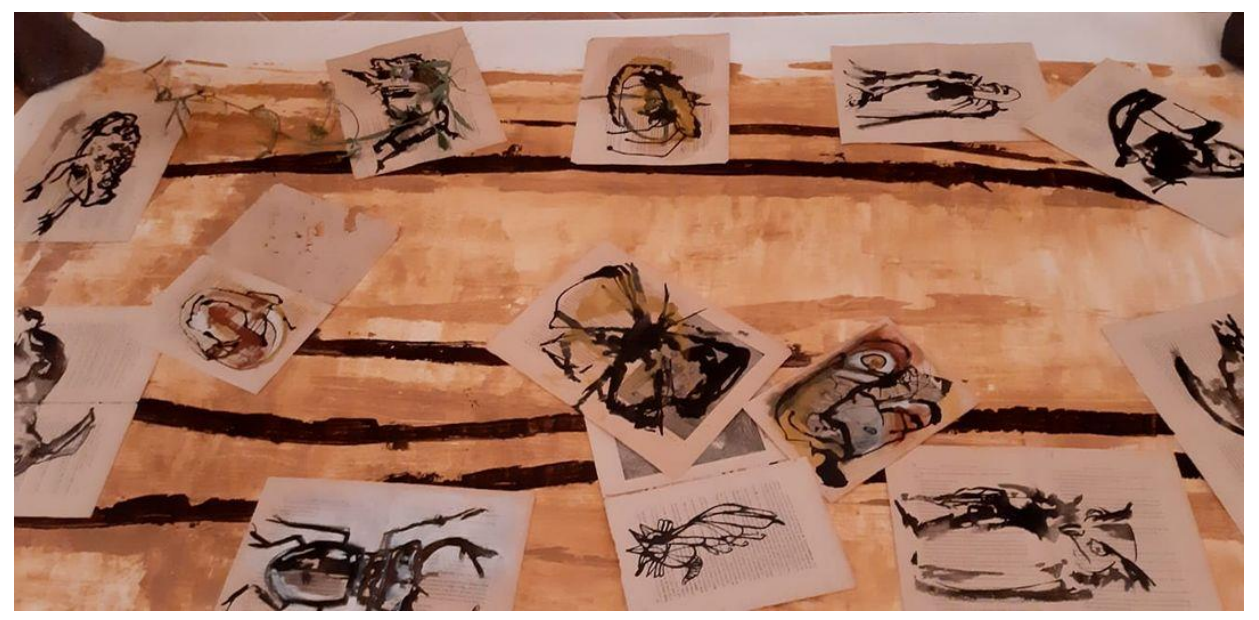

Oeuvres papier métamorphoses 202\& - Diane Cazelles

Je mène depuis cinq ans des exercices créatifs sur deux thèmes comparables, qui sont mis en scène (et en dessin) successivement avec les étudiants : « fusion Animal-Végétal » et «Viens chez moi, j'habite dehors ». Sur un temps assez court, un mois environ, les étudiants sont invités à réfléchir au végétal et à l'animal dont ils se sentent les plus proches et à les accoler ou les faire fusionner dans un dessin personnel. Ce dispositif proche des protocoles chamaniques relatifs aux «animaux de pouvoir » qui accompagnent l'humanité depuis les profondeurs du temps, représente un exercice de lien stimulant pour les jeunes adultes, leur ouvrant, par la même à un imaginaire créatif licite (une sorte de carte à jouer joker) et non plus enfoui dans une approche purement intime.

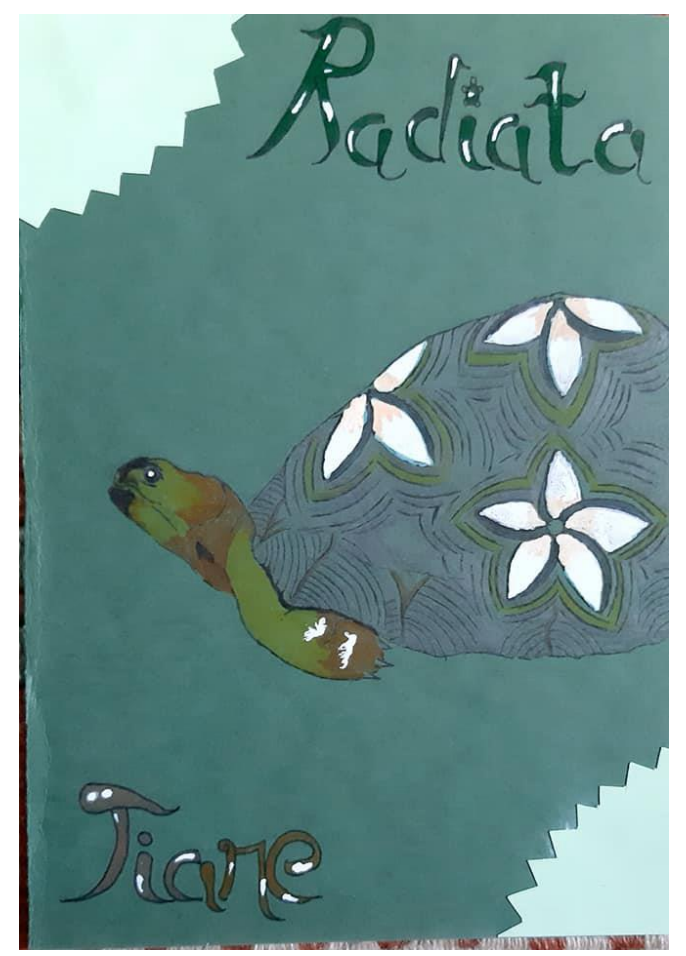

Matthieu A. Tortue Tiaré

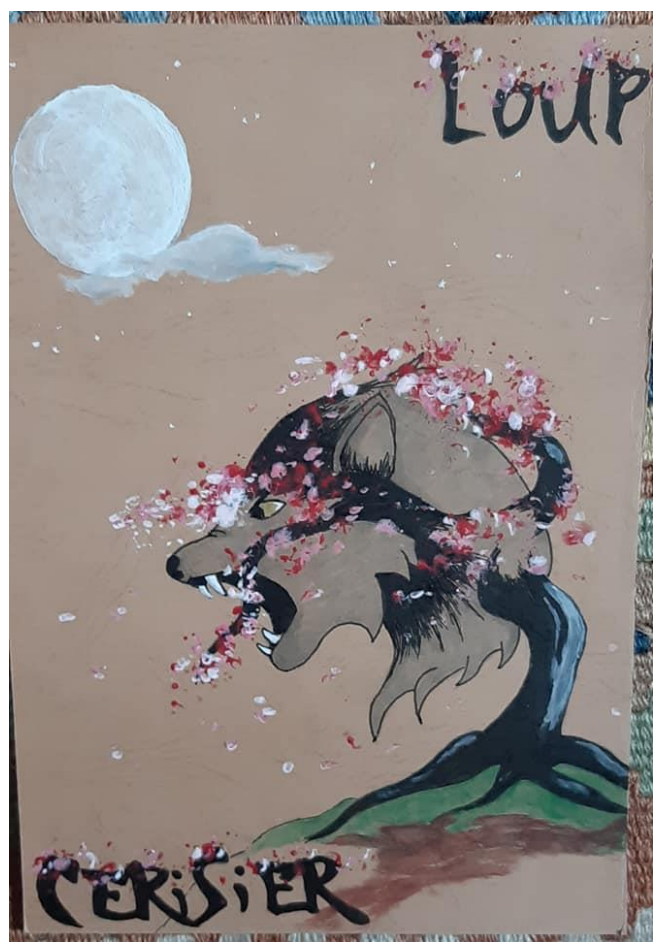

Danie Lancea-Loup cerisier, 2020

L'exercice qui suit « viens chez moi, j'habite dehors » correspond à la création d'un leporello de trois à cinq feuillets qui raconte, par le roman graphique qu'il met en scène, un entrainement impérieux vers un ailleurs. Si certains étudiants s'attachent à dessiner la rencontre d'un nanti avec un SDF qui l'oblige à sortir de sa zone de confort, d'autres convoquent des formes extraterrestres, au sens propre comme au sens figuré, parfois issues de l'être hybride précédent. Si les êtres d'appel sont plus souvent angéliques, c'est à dire brossés en traits de lumière et sans pieds, d'autres, par la 
maladresse du dessin ou l'audace même de la démarche inventive de l'étudiant, participent de la création d'un monde nouveau.

Citons encore, pour référence à notre propos, la réponse que le peintre Paul Klee fit en 1930 à un instituteur de Saxe qui souhaitait développer la créativité des enfants : "Menez vos élèves vers la nature, laissez-les voir comment un bourgeon se forme, comment un arbre grandit [...] La contemplation est une révélation, un aperçu de l'atelier de Dieu. Là-bas, dans la nature, repose le mystère de la création ». Comme les pédagogues de l'Antiquité l'avaient pensé, Aristote comme Héraclite, la contemplation attentive de «l'atelier de la nature » (=la biodiversité dans sa genèse) en fait accepter toutes les dérivations, les adaptations multiples que les gestes et les formes induisent. Pour Klee, la révélation a été celle de la lumière, celle de la couleur, puis celle du mouvement, trois réservoirs de la puissance de peindre, une trilogie agissante qui entraine l'artiste vers l'infini de la création..

Cette puissance concrète de la forme génésique s'incarne particulièrement dans l'œuvre organique de l'angevine Mélanie Pasquier ${ }^{5}$ dont la première exposition parisienne s'intitulait en 2018 «Un nouveau monde»: sa recherche explorait le cocon comme création toute à la fois, chaotique et ordonnée. Cette jeune peintre a, depuis la gestation et la naissance de son deuxième enfant, radicalement modifié son style pictural dont elle décrit ainsi la recherche originelle : « Entre traits et matière, un voyage sensoriel. Le point comme un atome, le corps de l'œuvre en gestation, fruit d'une lenteur nécessaire... ». Sa démarche qui allie le ressenti émotionnel avec les formes rondes, spores et les tiges du vivant offre une extraordinaire ouverture vers les formes extraterrestres, car issues de son corps et de la genèse imaginée de ce qui s'y déroule. La maternité comme expérience première des formes de l'avenir, secrètes, flottantes, plasmiques.

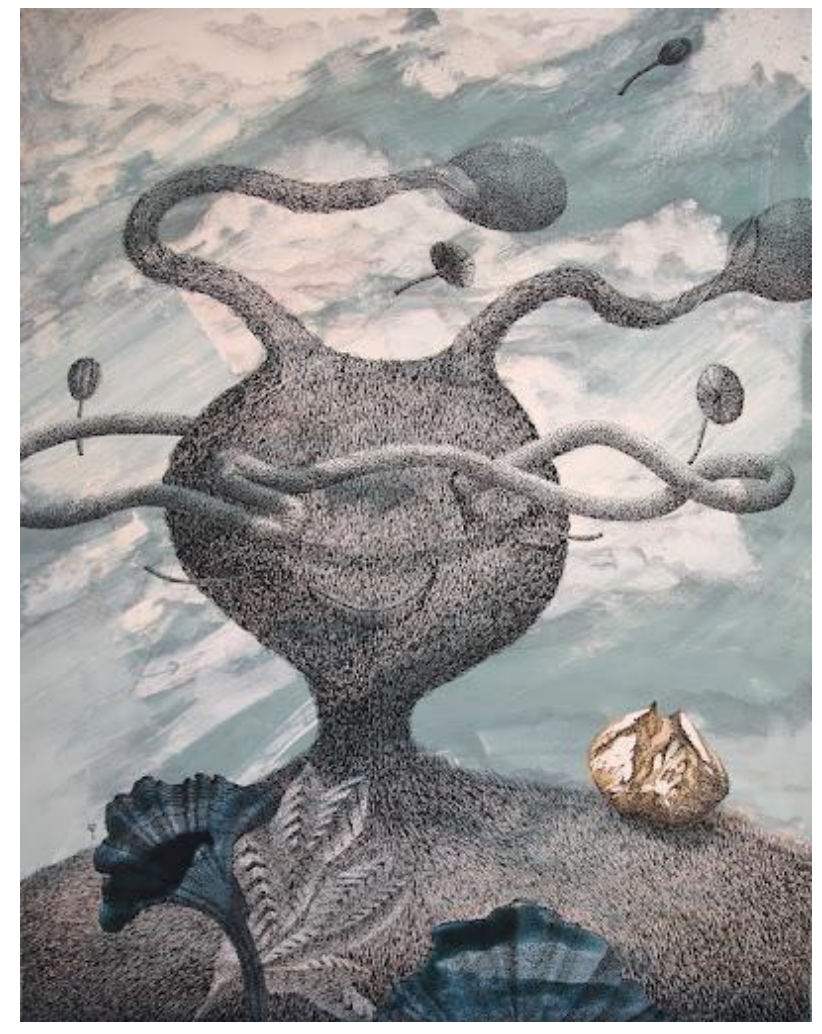

Mélanie Pasquier, Un nouveau Monde, 2018

\footnotetext{
${ }^{5}$ Mélanie Pasquier vit et travaille dans la région d'Angers. Cf. http://www.melaniepasquier.com
} 
La double démarche de l'essayiste Emmanuele Coccia (La Vie des plantes. Une métaphysique du mélange, 2016) et du romancier américain Richard Powers (L'arbre monde, 2018, Sidérations, 2021) doit stimuler cette réflexion sur les formes adaptatives du vivant lié à la photosynthèse. La métamorphose eucaryote, l'élan de la canopée au miroir de ses circuits racinaires, la communication des plantes, leur mise en sommeil et leur robustesse persévérante sont des éléments qui forcent l'admiration de ces deux auteurs du XXIeme siècle : ceux-ci opposent le génie évolutif des plantes et des arbres à notre ébriété numérique, ouvrant des pistes à des communications insoupçonnées. Le règne végétal est radicalement différent du règne des fungi (champignons) et, a fortiori, du règne animal dont nous sommes une sous-espèce. Bien peu d'entre nous peuvent en comprendre les modalités de la croissance végétale. Évolution, adaptation et croissance des organismes autotrophes : voici le terrain mal exploré des existences extra-terrestres, dans la mesure où les graines ont besoin de l'air et des autres organismes pour s'échapper de l'emprise racinaire.

Genèse et germination sont les deux pistes sur lesquelles les artistes ont la part belle, au delà des chimères que l'hybridation anthropocentrique peut imaginer. E. Coccia qui pense l'enveloppe gazeuse de l'atmosphère comme le mélange originel exprime ainsi sa fascination végétale «les plantes sont le souffle de tous les êtres vivants, le monde en tant que souffle. »Et Richard Powers de citer Rûmi : "l'amour est un arbre aux branches dans le toujours/aux racines dans l'éternité/Au tronc nulle part». Et si chaque tronc élancé vers le ciel dépend d'une communauté secrète, peutêtre que les extra-terrestres que nous tentons d'imaginer sont déjà implantés dans l'humus depuis des millions d'années...

\section{Bibliographie}

Sylvie DALLET, Georges CHAPOUTHIER, Émile NOËL (dir.), La création, définitions et défis contemporains, collection «Éthiques de la Création» (Harmattan/ Institut Charles Cros), 2009

Sylvie DALLET \& Eric DELASSUS (dir.), Éthiques du goût, collection «Éthiques de la Création » (Harmattan/ Institut Charles Cros), 2014

Roger GARAUDY, Esthétique et invention du futur, 10/18, 1971

Gérard HOLTON, L'imagination scientifique, Gallimard, traduction 1981

Jean-Pierre JOST \& Yan Chim JOST-TSE : L'automédication chez les animaux dans la nature et ce que nous pourrions apprendre d'eux (Éditions Connaissances \& Savoirs, 2015)

Richard POWERS, L'arbre-monde, éditions du Cherche midi, 2018 et Sidérations, Actes Sud, 2021

Pascal QUIGNARD, Abîmes, Grasset, 2002

Paul VIRILIO, Vitesse et politique, Galilée, 1977

\section{Articles}

Sylvie DALLET, «Le mondes est tel qu'il est, mais nous, où sommes nous ? », in Savoirs de frontières (Image, écriture, oralité), collectif sous la direction de Sylvie Dallet \& Élie Yazbek, collection «Éthiques de la Création » (Harmattan/ Institut Charles Cros), 2013

Sylvie DALLET «L'Avenir à reculons : genre et gens de la caverne », in le mythe de la caverne aujourd'hui-Ce que Platon dit de nous..., coordination Rémi Astruc \& Alexandre Georgandas, Ellipses, 2015

\section{Films}

Werner HERZOG, La grotte des rêves perdus, 2010

Chris MARKER, Immemory, 1997 